\title{
Recognition of human face images by the free flying wasp Vespula vulgaris
}

\author{
Aurore Avarguès-Weber ${ }^{1 \dagger}$, Daniele d'Amaro ${ }^{2 \dagger}$, Marita Metzler $^{2 \dagger}$, Jair E. Garcia ${ }^{3}$ \\ and Adrian G. Dyer ${ }^{3,4 *}$ \\ ${ }^{1}$ Centre de Recherches sur la Cognition Animale, Centre de Biologie Intégrative (CBI), Université de Toulouse, CNRS, UPS, \\ Toulouse, France \\ ${ }^{2}$ Institut für Zoologie III (Neurobiologie), Johannes Gutenberg-Universität, Mainz, Germany \\ ${ }^{3}$ Royal Melbourne Institute of Technology, Melbourne, VIC, Australia \\ ${ }^{4}$ Monash University, Clayton, VIC, Australia \\ $\dagger$ These authors contributed equally \\ *Corresponding author (Email: adrian.dyer@rmit.edu.au)
}

Citation - Avarguès-Weber, A., d'Amaro, D., Metzler, M., Garcia, J. E., \& Dyer, A. G. (2017). Recognition of human face images by the free flying wasp Vespula vulgaris. Animal Behavior and Cognition, 4(3), 314-323. https:// doi.org/10.26451/abc.04.03.09.2017

\begin{abstract}
The capacity to recognize perceptually similar complex visual stimuli such as human faces has classically been thought to require a large primate, and/or mammalian brain with neurobiological adaptations. However, recent work suggests that the relatively small brain of a paper wasp, Polistes fuscatus, possesses specialized face processing capabilities. In parallel, the honeybee, Apis mellifera, has been shown to be able to rely on configural learning for extensive visual learning, thus converging with primate visual processing. Therefore, the honeybee may be able to recognize human faces, and show sophisticated learning performance due to its foraging lifestyle involving visiting and memorizing many flowers. We investigated the visual capacities of the widespread invasive wasp Vespula vulgaris, which is unlikely to have any specialization for face processing. Freely flying individual wasps were trained in an appetitive-aversive differential conditioning procedure to discriminate between perceptually similar human face images from a standard face recognition test. The wasps could then recognize the target face from novel dissimilar or similar human faces, but showed a significant drop in performance when the stimuli were rotated by $180^{\circ}$, thus paralleling results acquired on a similar protocol with honeybees. This result confirms that a general visual system can likely solve complex recognition tasks, the first stage to evolve a visual expertise system to face recognition, even in the absence of neurobiological or behavioral specialization.
\end{abstract}

Keywords - Hymenoptera, Individual recognition, Insect vision, Visual learning

The capacity of animals to remotely identify other individuals using visual cues can convey important fitness benefits by avoiding conflicts, or strengthening social bonds (Haxby, Hoffman, \& Gobbini, 2002; Tibbetts \& Dale, 2007). Humans are experts at face recognition (Carey, De Schonen, \& Ellis, 1992; Mondloch, Maurer, \& Ahola, 2006; Tanaka \& Gauthier, 1997; Yin, 1969), a capacity that is facilitated by specialized regions like the Fusiform Face Area (FFA) located in the fusiform gyrus, and associated regions in the brain (Kanwisher, 2000; Kanwisher, McDermott, \& Chun, 1997; Zhen, Fang, \& Liu, 2013). Our specialization for face processing is already evident even at childbirth (de Haan, Pascalis, \& Johnson, 2002; Johnson, Dziurawiec, Ellis, \& Morton, 1991; Shah, Happé, Sowden, Cook, \& Bird, 2015; Turati, Bulf, \& Simion, 2008), although visual experience allows subsequent perceptual and 
neurobiological tuning (de Haan et al., 2002; Pascalis, de Haan, \& Nelson, 2002). Face specialization is also evident in other primate species that also have dedicated neural circuitry for face processing (Rosenfeld \& Van Hoesen, 1979; Tsao, Freiwald, Tootell, \& Livingstone, 2006), as well as by some other mammals such as sheep (Kendrick, Costa, Leigh, Hinton, \& Peirce, 2001).

Face expertise is often facilitated by a dedicated visual mechanism, termed holistic or configural processing, allowing very efficient recognition despite sometimes subtle perceptual differences between faces, or variations in viewpoint orientation (Carey \& Diamond, 1977; Maurer, Le Grand, \& Mondloch, 2002; Peterson \& Rhodes, 2003; Tanaka \& Sengco, 1997; Young, Hellawell, \& Hay, 1987). This specific processing consists of analyzing the spatial relations among facial features and seems to require in mammals specific brain structures acquired through evolution (Kanwisher, 2000; Maurer et al., 2002), although other vertebrates like pigeons (Stephan, Wilkinson, \& Huber, 2012), crows (Marzluff, Miyaoka, Minoshima, \& Cross, 2012) or archerfish (Newport, Wallis, Reshitnyk, \& Siebeck, 2016) appear to also be able to very reliably discriminate and recognize human faces. The proposal that a large brain is necessary for face processing and individual recognition has been challenged by evidence that an insect, the wasp Polistes fuscatus, can individually recognize nest mates from their facial visual mask (Tibbetts, 2002).

Polistes fuscatus queens typically join forces and live in a nest in which a hierarchy is established through a series of sequential combats between two dueling individuals, from which the strongest wasp becomes queen and dominates reproduction, whilst subordinate wasps do more rudimentary tasks within the hive. Individual Polistes fuscatus wasps recognize their previous opponents by distinctive facial markings to avoid the repetition of potentially costly battles (Tibbetts, 2002). These wasps also showed enhanced discrimination abilities between images of conspecifics' faces in an aversive differential conditioning task incorporating a T-shaped maze with a mild aversive electric shock for incorrect choices to the negatively reinforced conditioned visual stimulus (CS-). In comparison, the choice of the alternative 'correct' stimulus received no reinforcement (Sheehan \& Tibbetts, 2011). This face specialization in individual Polistes fuscatus wasps was significantly enhanced compared to visual processing for other complex pictures like prey items or even simple geometric patterns (Sheehan \& Tibbetts, 2011). Interestingly, the level of performance dropped if the wasp face stimuli were manipulated through antennae removal or picture scrambling (Sheehan \& Tibbetts, 2011). These results are therefore consistent with the existence of face expertise in the miniaturized invertebrate brain of Polistes fuscatus, even if potential dedicated mechanisms such as configural processing or brain specialization currently remain to be confirmed in this species (Avarguès-Weber, 2012; Chittka \& Dyer, 2012; Tibbetts \& Dyer, 2013).

In parallel, the honeybee has emerged as a major model of visual cognitive processing in insects (Avarguès-Weber, Deisig, \& Giurfa, 2011; Avarguès-Weber, Dyer, Combe, \& Giurfa, 2012; AvarguèsWeber, Dyer, Ferrah, \& Giurfa, 2015; Avarguès-Weber \& Giurfa, 2013; Chittka \& Niven, 2009; Howard, Avarguès-Weber, Garcia, \& Dyer, 2017; Srinivasan, 2010). The honeybee can be trained to solve perceptually or cognitively difficult visual tasks in free-flying conditions by collecting sucrose associated with a target visual stimulus while an alternative stimulus offers a distasteful substance (quinine; AvarguèsWeber, de Brito Sanchez, et al., 2010). Honeybees are very amenable to visual cognitive studies as individuals collect nutrition for the entire colony, returning to the hive to offload the food collected when satiated, thus retaining an intact motivation for the sucrose reward throughout the entire coarse of a day, enabling consequently long training and associated testing to understand the mechanisms underlying visual perception (Avarguès-Weber et al., 2015; Avarguès-Weber, Portelli, et al., 2010; Dyer \& Griffiths, 2012; Gross et al., 2009; Stach \& Giurfa, 2005). It has now been well established that individual bees can learn to recognize complex pictures, such as human faces (Dyer, Neumeyer, \& Chittka, 2005; Dyer \& Vuong, 2008). They are also capable of categorizing visual stimuli on the basis of painting style (Wu, Moreno, Tangen, \& Reinhard, 2012), geometric properties (Benard, Stach, \& Giurfa, 2006; Giurfa, Eichmann, \& Menzel, 1996; Horridge \& Zhang, 1995; van Hateren, Srinivasan, \& Wait, 1990) or natural landscapes (Dyer, Rosa, \& Reser, 2008; Zhang, Srinivasan, Zhu, \& Wong, 2004). The impressive visual learning abilities by honeybees could be explained by the fact that these pollinators often rely on the complex visual signals of flowers to acquire food. For example, bees may need to memorize complex flower patterns to avoid deceptive plant flowers (Stejskal, Streinzer, Dyer, Paulus, \& Spaethe, 2015). Interestingly, it has also 
been demonstrated that honeybees have the capacity to rely on configural processing with prolonged visual experience (Avarguès-Weber, Portelli, et al., 2010; Stach, Benard, \& Giurfa, 2004; Stach \& Giurfa, 2005), thus offering a fascinating parallel with primate visual processing (Avarguès-Weber et al., 2015).

To develop further insights into how animals can recognize complex pictures, such as faces, it is of high comparative value to assess the abilities of other insect species. We therefore tested a wasp species that could be intensively trained in similar free flying conditions to honeybees, and which has a low likelihood of having face processing expertise. The common European wasp Vespula vulgaris is a widespread invasive species that appears adaptable to many environments, and is a food generalist (Archer \& Penney, 2012; Beggs et al., 2011; Grangier \& Lester, 2012). These wasps live in colonies founded by a single queen, and dwell in enclosed ground nests that would offer limited lighting conditions for individual recognition by visual cues (Archer, 2010). This species does not have the selective pressure to develop individual recognition as has been demonstrated for Polistes fuscatus wasps (Tibbetts, 2002). Despite these converging evidentiary elements, we additionally investigated the possibility of individual face recognition in Vespula vulgaris by analyzing the variance in facial features of these wasps using the methodology developed by Sheehan and Tibbetts (2010). We then developed an appetitive-aversive free flying differential conditioning paradigm for Vespula vulgaris to test if this wasp can learn and accurately discriminate images of human faces by employing the method and stimuli as has been used previously to demonstrate the recognition of human faces by free-flying honeybees (Avarguès-Weber, Portelli et al., 2010; Dyer et al., 2005).

\section{Method}

\section{Variability in Facial Features of Vespula vulgaris Wasps}

Experiments were conducted at Johannes Gutenberg-Universität, Mainz, Germany in late summer when Vespula vulgaris wasps could be found visiting gravity feeders filled with sucrose solution (12\% (vol/vol)). The wasps feeding at the gravity feeder were visually tracked to find their nest that was established about $35 \mathrm{~m}$ away from our test site. We collected a total of 26 wasps around the nest. A digital camera was used to capture the facial features of each of these wasps. We were specifically interested in the clypeus region of the wasp face as this region shows high variability for wasps (Polistes fuscatus) demonstrating individual recognition but low variability in a relative wasp species (Polistes dominulus) that do not exhibit individual recognition (Sheehan \& Tibbetts, 2010). The clypeus region of each individual Vespula vulgaris wasp was isolated with Photoshop selection tools, converted to achromatic images by equalizing the output of the red, green and blue color channels and image segmentation was conducted using threshold values to create binary images representing either the 'black' or 'yellow' components of the wasp clypeus (Figure 1A). The respective surface areas were converted to percentage area coverage following the methodology of Sheehan and Tibbetts (2010).

\section{Training Procedure}

The wasps were individually tagged and trained to freely visit the experimental apparatus offering a $25 \%$ sucrose solution. Our apparatus was a grey circular plastic vertical screen $(50 \mathrm{~cm}$ in diameter) that could be rotated to vary the spatial arrangement of the stimuli presented on freely rotating hangers (Dyer et al., 2005, Figure 2A). Twelve wasps completed the entire training and testing procedure and were kept for analysis. Only one wasp was present at a time at the apparatus during the training and the tests. Four achromatic human faces stimuli (two identical S+ and two identical S- stimuli; Figure 2A, B) taken from standard face recognition tests (Warrington, 1996) were presented simultaneously above the horizontal landing platforms, which each offered a $5 \mu \mathrm{L}$ drop of either a $25 \%(\mathrm{vol} / \mathrm{vol})$ sucrose solution $(\mathrm{S}+)$ or a 60 $\mathrm{mM}$ quinine hemisulfate solution ( $\mathrm{S}-$ ). The use of quinine solution promotes enhanced visual discrimination performances in other hymenopteran insects including honeybees and bumblebees (Avarguès-Weber, de 
Brito Sanchez, et al., 2010; Chittka, Dyer, Bock, \& Dornhaus, 2003; Rodríguez-Gironés, Trillo, \& Corcobado, 2013).
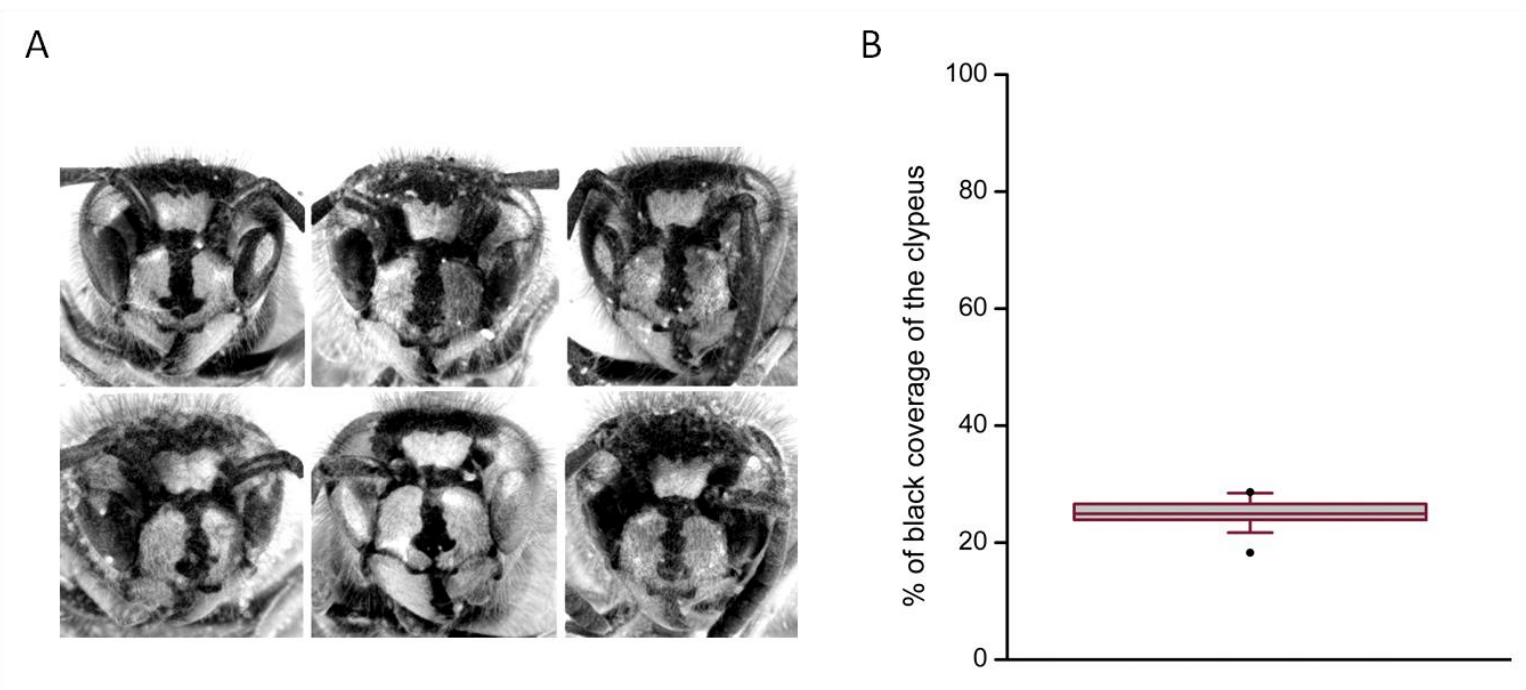

Figure 1. A) Pictures of six representative Vespula vulgaris wasps showing that key face visual features like the clypeus show little variation between individuals. B) Box plot of black clypeus coverage expressed as a percentage of total clypeus surface area of Vespula vulgaris wasp faces $(N=26)$. The whisker bars indicate $5 \%$ and $95 \%$ confidence intervals and the dots show the minimal and maximal values.

The face stimuli were attached on the $6 \times 8$-cm hangers that could be positioned in a number of random spatial positions and rearranged during the training when the test wasp was absent either when returning to its nest or after a $\mathrm{S}+$ choice by a rotation of the whole screen or manual displacements of the hangers (Figure 2A). Indeed, when a wasp landed on a S+ platform and drank the sucrose drop, an additional drop was presented on a transparent Plexiglas spoon so the wasp could be placed behind an opaque screen while the screen was rotated. The wasp was then allowed to approach the apparatus again to make a novel choice. This method was developed to promote learning of complex patterns including faces in free flying honeybees (Avarguès-Weber, Portelli, et al., 2010; Dyer et al., 2005, 2008; Dyer \& Vuong, 2008; Stejskal et al., 2015). This procedure avoided positional learning of the rewarding stimuli and ensured that the stimuli could be visually inspected between choices. Before returning to the nest to deliver the imbibed sucrose to nest-mates, the wasps made typically $5-6$ choices (landing on a stimulus platform). The wasps were fast (typically $1-2 \mathrm{~min}$ ) between finishing a bout and returning from delivering collected nutrition to the nest. The training continued for a total of 90 choices, and the whole training session lasted approximately three hours per individual wasp. Stimuli and landing platforms were washed with $20 \%$ ethanol solution and then fresh water between foraging bouts, and before each of the tests. In addition, fresh stimuli were used in the non-reinforced tests.

\section{Tests}

Following training, five non-reinforced test conditions were presented to the wasps. In each test, the first 20 choices were recorded. The respective test sessions were intermingled with refreshing foraging bouts presenting the training conditions ( $\mathrm{S}+$ and $\mathrm{S}$ - stimuli reinforced respectively with sucrose and quinine solutions) until the wasp made $10 \mathrm{~S}+$ choices $(1-3$ foraging bouts). This maintained wasp motivation. Each wasp was presented with a learning test presenting the training stimuli allowing for the assessment of $\mathrm{S}+/ \mathrm{S}$ - discrimination level after the training session (Learning test; Figure $2 \mathrm{~B}$ ). Then, three different nonreinforced tests followed to investigate further visual processing by the wasps by presenting the $S+$ face against different distracters that varied in their level of perceptual similarity (assessed by human ranking, 
see Dyer et al., 2005, for details) from the S+ (Test a: schematic representation of a face, Test $b$ and Test $c$ : novel face pictures; Figure 2B). A final test consisted of offering a choice between $180^{\circ}$ rotated version of the S+ and S- faces (Inverted test; Figure 2B). The different tests were presented in a random sequence order for each wasp.

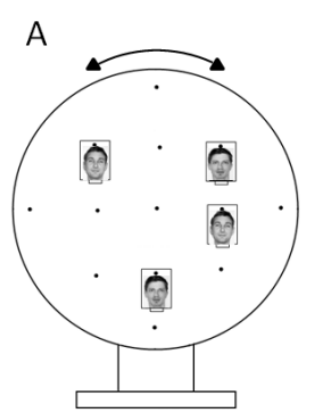

B
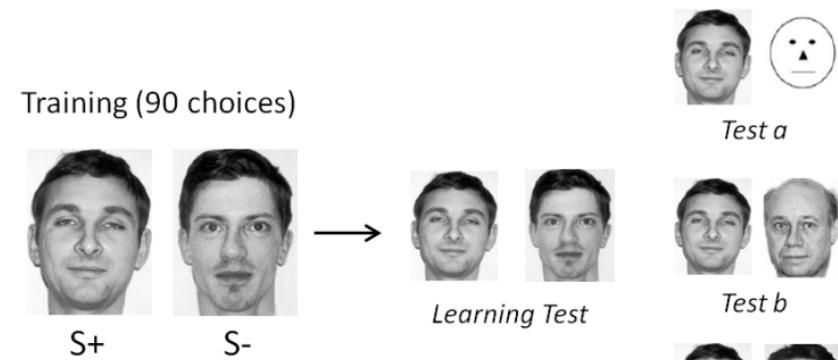

Test a
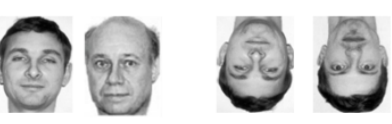

Test b

Inverted Test

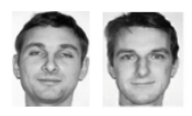

Test c
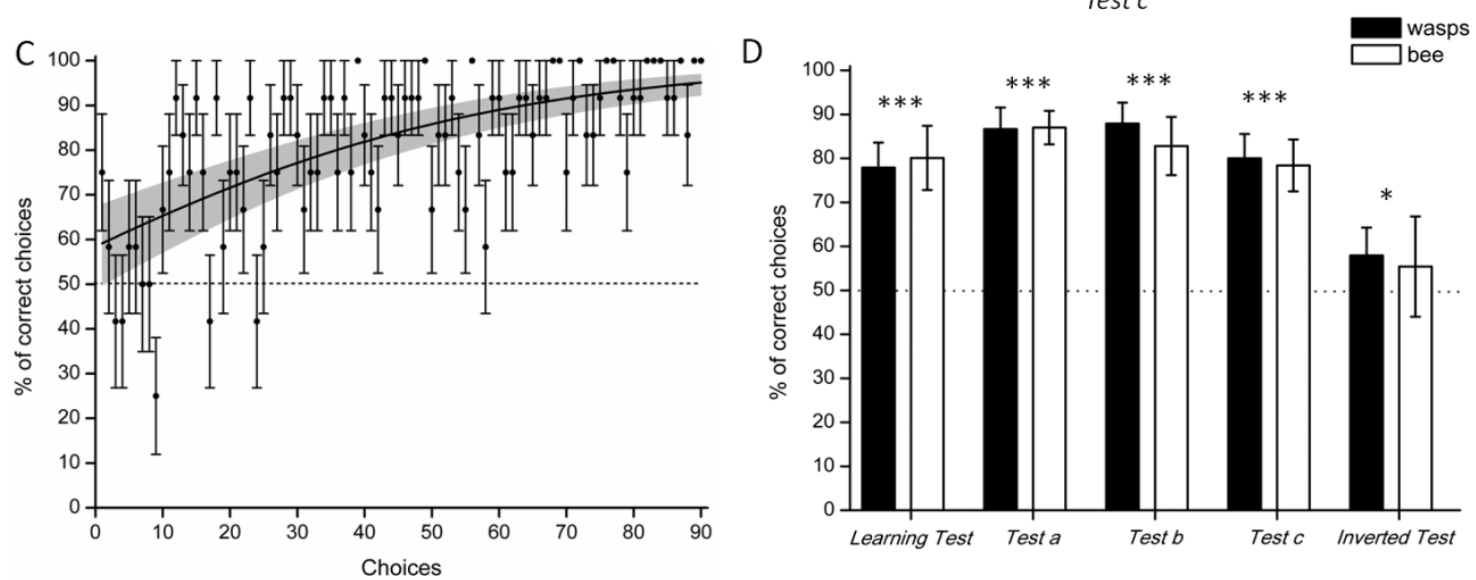

Figure 2. A) Schematic representation of the experimental setup. B) Stimuli used for training and the non-reinforced tests. C) Mean percentage of choices for the $\mathrm{S}+$ stimulus in each of the 90 consecutive choices. The solid curve represents the linear fit model with 95\% confidences intervals (shaded area) while the points indicate the data collected from the wasps $(\mathrm{N}=12$, mean \pm S.E.M.). D) Performances in the five different non-reinforced tests in terms of percentage of correct choices (mean $\pm 95 \%$ C.I.) on the 20 total test choices. The dashed line indicates chance level. The honeybees' data (white bars) were reproduced from (Dyer et al., 2005) to allow a qualitative comparison between Vespula vulgaris wasps and Apis mellifera honeybees' performances. The experimental procedures as well as training and testing stimuli were similar for both species. ${ }^{* * *} p<0.001 . * p<0.05$.

\section{Statistical Analysis}

Acquisition performances were analyzed using a generalized linear mixed model (GLMM, R 3.3.3 (R Core team), lme4 package). The response variable consisted of binary choices (S+ or S-) for each wasp modeled assuming a binomial distribution with a logit link function. The choice number was included as a fixed factor and individual wasps were considered as a random factor to account for the repeated measurement design. Performances during the tests (proportion of correct choices out of the 20 test choices; a single value by wasp) were analyzed with a generalized linear model (GLM) selecting a binomial distribution and a logit link function. This model included only the intercept term to test for a significant difference between the mean proportion of observed correct choices $(p)$ and the proportion of choices expected by chance $(p=0.5)$. Finally, the performances of the four transfer tests (Figure 2B) were compared to the performance level of the learning test with a GLM including the type of test as a categorical variable with five levels. 


\section{Results}

\section{Variability in Facial Features of Vespula vulgaris Wasps}

The analysis of the percentage of black in the clypeus in $26 \mathrm{~V}$. vulgaris wasps (mean: $25.0 \%$, min: 18.2\%, max: 28.6\%) shows a low amount of individual variation (S.E.M: 0.44\%) (Figure 1B), suggesting a low likelihood of conspecific individual recognition according to the framework provided by Sheehan and Tibbetts (2010). The variance is indeed lower than in the wasp P. dominulus that lacks individual recognition (individual variation between 0 and 30\%, see (Sheehan \& Tibbetts, 2010).

\section{Discrimination of Human Faces Pictures}

Wasps $(n=12)$ succeeded in solving the discrimination task between the two human faces pictures (S+ vs. S-; Figure 2B) as choices for the rewarded stimulus significantly increased across choices $(\mathrm{z}=8.47$, $p<0.001$; Figure 2C).

Performance from the learning test (S+ vs. S-) confirmed that the wasps were able to master the discrimination learning task (mean proportion: 77.9\%, 95\% CI: 72.2, 82.7; $\mathrm{z}=10.0, p<0.001$; Figure 2D).

The wasps also recognized the target stimulus when alternative stimuli were used as distractors. The wasps preferred the S+ stimulus against a highly schematic representation of a face (Test a: $86.7 \%$, CI: $81.8,90.4 ; \mathrm{z}=9.86, p<0.001$; Figure 2D) or against the two novel faces that varied in their respective level of perceptual similarity with the $\mathrm{S}+$ stimulus (Test $b: 87.9 \%, \mathrm{CI}: 83.2,91.5 ; \mathrm{z}=10.0, p<0.001$; Test $c: 80.0 \%$, CI: 74.5, 84.6; $\mathrm{z}=8.59, p<0.001$; Figure 2D). The level of discrimination performance was significantly higher when compared to the results from the learning test if the schematic face (Test $a: \mathrm{z}=$ $2.49 ; p=0.01$; Figure 2D) or the most dissimilar face (Test $b: \mathrm{z}=2.87 ; p=0.004$; Figure 2D) were proposed as alternatives from the S+. However, the performances were comparable to the learning test and when a similar face was used as distractor (Test $c: \mathrm{z}=0.56 ; p=0.58$; Figure 2D).

The wasps, however, had significantly greater difficulty to recognize a $180^{\circ}$ rotated version of the S+ stimulus (Inverted test: $57.9 \%, \mathrm{CI}: 51.6,64.0 ; \mathrm{z}=2.44, p=0.02$; comparison to learning test: $\mathrm{z}=4.63$; $p<0.001$; Figure 2D), as only four wasps out of the 12 tested preferred the inverted S+ stimulus (> $55 \%$ of correct choices); whereas all wasps were successful in all of the other tests.

The performances of the wasps were comparable to the performances of the honeybees tested in Dyer et al. (2005), Figure 2D, white bars).

\section{Discussion}

We assessed the capacity of Vespula vulgaris wasps to learn images of human faces from a standard test (Warrington, 1996) used to quantify face recognition in human subjects. The wasps in a free flying condition were able to learn to approach a target stimulus to collect a reward and to avoid a similar distractor associated with a distasteful substance thus following an appetite-aversive differential conditioning procedure known to improve visual learning in other hymenoptera (Avarguès-Weber, de Brito Sanchez, et al., 2010; Chittka et al., 2003; Rodríguez-Gironés et al., 2013). The wasps were then able to discriminate between the training face stimuli and subsequently to recognize the target face when presented against completely novel faces.

Qualitatively, the performance of the wasps showed a very similar pattern to the performance of honeybees with these same face stimuli as revealed in a previous study (Dyer et al., 2005; Figure 2D). Because the converging evidence suggest that these wasps are unlikely to possess specialized face processing (see Introduction and Figure 1), these findings confirm that a miniature brain of a hymenopteran insect can learn very complex but completely novel patterns like face images without any biological relevance associated. This conclusion suggests that the first stages to develop, or evolve, face processing expertise may not be challenging for animals already possessing complex visual learning for foraging or navigation. Indeed, there have been many reports of different animal species recognizing either conspecifics 
(Tibbetts \& Dale, 2007) or even human faces (Marzluff et al., 2012; Newport et al., 2016; Stephan et al., 2012). The acquisition of facial pattern recognition in paper wasps may consequently be promoted from visual learning abilities if the appropriate visual information is available. Thus, in the hover wasp, Liostenogaster flavolineata (Baracchi, Turillazzi, \& Chittka, 2016), the facial pattern variability correlates with colony membership, thus enabling reliable visual categorization of relevant social groups. These wasps were indeed shown to prioritize visual over olfactory cues to fight against intruders (Baracchi, Petrocelli, Chittka, Ricciardi, \& Turillazzi, 2015).

Our finding that a relatively small and simple wasp brain can recognize very complex visual stimuli representing human faces does not, however, necessarily mean that other insects with a functional visual system may possess such a perceptual capacity. Hymenopteran insects, including bees and wasps, do show particular brain specificities compared to other insects, such as the classical diptera insect model Drosophila, which could be linked to sophisticated visual abilities (Chittka \& Niven, 2009; Farris, 2008). In particular, the brains of hymenoptera possess relatively large and elaborate mushroom bodies where areas like the calyces, receiving afferences from primary visual centers, are doubled and expanded in relation to the lobes (Chittka \& Niven, 2009; Farris, 2008). Such adaptation in this insect order, including all social insect species, has led to the appealing transposition of the 'social brain' hypothesis developed as a potential explanation of brain development in mammals (Dunbar \& Shultz, 2007) to social insects (Lihoreau, Latty, \& Chittka, 2012). However, the relative development of the calyces and mushroom body in insects started back with ancestral parasitoid wasps that already possessed spatial, visual or olfactory learning facilities (Farris \& Schulmeister, 2011; Lihoreau et al., 2012). Interestingly, when Gronenberg, Ash, and Tibbetts (2008) tested brain size and neuroanatomy of four different species of wasps, including species that demonstrate facial pattern recognition or lacked such a capacity, there was no discernible difference in respective optic lobe size. However, there was some evidence that subtle mushroom body variability between species may be correlated with face recognition abilities. Given the absence of difference at the primary visual centers (optic lobes), the authors suggested that the brain of these four species might be pre-adapted for pattern discrimination, and that the ability to discriminate facial markings might require only small modifications to underlying neuronal substrates at the mushroom bodies level (Gronenberg et al., 2008), potentially even occurring within the lifespan of an individual insect due to experience-dependent plasticity. The social brain hypothesis proposed to account for enlarged mushroom bodies in social insects (Lihoreau et al., 2012) might thus be replaced by an adaptive role of mushroom bodies to allow for an increase in learning and flexibility, consequently enabling visual cognitive processes previously thought to be the domain of higher vertebrates.

The development of the mushroom bodies may also play a role in the capacity to use configural processing to reliably recognize complex visual stimuli. Configural and holistic processing may indeed require analysis at a higher-order than simple feature recognition, as the topological information also needs to be acquired. Some initial evidence suggests that the visual individual recognition capacity of Polistes fuscatus wasps may rely on the processing of spatial relations between face features because the recognition performance is impaired by simulus manipulation thought to alter configural information (Sheehan \& Tibbetts, 2011). Definitive proof of configural processing in insects has, however, so far been demonstrated only in honeybees (Avarguès-Weber, Portelli, et al., 2010). In our current study, a significant drop of performance by Vespula vulgaris wasps was observed when the stimuli were rotated by $180^{\circ}$, a manipulation that also potentially disssrupts configural information, consistent with previous honeybee results (Avarguès-Weber, Portelli, et al., 2010; Dyer et al., 2005; Figure 2D). This is suggestive that configural or holistic processing might be shared by other hymenopteran species.

The question of whether other insect species could rely on configural processing for a higher recognition efficiency of complex images different to natural stimuli that might be typically encountered in a forager's life is fascinating from a comparative perspective. This would be indeed a beautiful example of evolutionary convergence with primate visual processing. Some hymenopteran insects are ideal candidates for future comparative studies. For example, bumblebees, which also typically visit many flowers in complex environments, are already known to be able to learn various visual parameterized stimuli and complex images as reliable representations of real flowers (Essenberg, Easter, Simmons, \& Papaj, 2015; 
Nityananda, Skorupski, \& Chittka, 2014; Thompson \& Plowright, 2014; Wolf, Roper, \& Chittka, 2015). Another example could be an ant species, Gigantiops destructor, which relies on visual memory to navigate in its tropical forest natural habitat while also being able to use visual cues as indicators to navigate in artificial mazes (Beugnon, Chagné, \& Dejean, 2001; Wystrach \& Beugnon, 2009). Our demonstration that it is possible to recruit and train Vespula vulgaris wasps to participate in free flying appetitive-aversive differential conditioning experiments enabling extensive visual training and testing should hopefully open the door for the potential testing of other central place foraging species under analogous conditions.

\section{Acknowledgements}

We are grateful for advice and assistance provided by Dr Jürgen Schramme and Professor Roland Strauss to help facilitate this research. AGD acknowledges the Australian Research Council DP0878968/DP0987989 and the Alexander von Humboldt Foundation for assistance in developing this work.

\section{References}

Archer, M. (2010). The queen colony phase of vespine wasps (Hymenoptera, Vespidae). Insect Society, 57, 133 - 145. Archer, M. E., \& Penney, D. (2012). Vespine wasps of the world: Behaviour, ecology and taxonomy of the Vespinae. Castleton, UK: Siri Scientific Press.

Avarguès-Weber, A. (2012). Face recognition: Lessons from a wasp. Current Biology, 22, R91 - R93.

Avarguès-Weber, A., de Brito Sanchez, M. G., Giurfa, M., \& Dyer, A. G. (2010). Aversive reinforcement improves visual discrimination learning in free-flying honeybees. PLOS ONE, 5, e15370.

Avarguès-Weber, A., Deisig, N., \& Giurfa, M. (2011). Visual cognition in social insects. Annual Review of Entomology, 56, $423-443$.

Avarguès-Weber, A., Dyer, A. G., Combe, M., \& Giurfa, M. (2012). Simultaneous mastering of two abstract concepts with a miniature brain. Proceedings of the National Academy of Sciences, USA, 109, $7481-7486$.

Avarguès-Weber, A., Dyer, A. G., Ferrah, N., \& Giurfa, M. (2015). The forest or the trees: Preference for global over local image processing is reversed by prior experience in honeybees. Proceedings of the Royal Society, B, 282, 20142384.

Avarguès-Weber, A., \& Giurfa, M. (2013). Conceptual learning by miniature brains. Proceedings of the Royal Society of London B, 280, 20131907.

Avarguès-Weber, A., Portelli, G., Benard, J., Dyer, A. G., \& Giurfa, M. (2010). Configural processing enables discrimination and categorization of face-like stimuli in honeybees. Journal of Experimental Biology, 213, $593-601$.

Baracchi, D., Petrocelli, I., Chittka, L., Ricciardi, G., \& Turillazzi, S. (2015). Speed and accuracy in nest-mate recognition: A hover wasp prioritizes face recognition over colony odour cues to minimize intrusion by outsiders. Proceedings of the Royal Society, B, 282. 20142750.

Baracchi, D., Turillazzi, S., \& Chittka, L. (2016). Facial patterns in a tropical social wasp correlate with colony membership. The Science of Nature, 103, 80. doi:10.1007/s00114-016-1406-8.

Beggs, J. R., Brockerhoff, E. G., Corley, J. C., Kenis, M., Masciocchi, M., Muller, F., Rome, Q., \& Villemant, C. (2011). Ecological effects and management Vespidae of invasive alien. BioControl, 56, 505. doi: 10.1007/s10526-011-9389-z

Benard, J., Stach, S., \& Giurfa, M. (2006). Categorization of visual stimuli in the honeybee Apis mellifera. Animal Cognition, 9, $257-270$.

Beugnon, G., Chagné, P., \& Dejean, A. (2001). Colony structure and foraging behavior in the tropical formicine ant, Gigantiops destructor. Insect Society, 48, $347-351$.

Carey, S., De Schonen, S., \& Ellis, H. (1992). Becoming a face expert [and discussion]. Philosophical Transactions of the Royal Society B, 335, $95-103$.

Carey, S., \& Diamond, R. (1977). From piecemeal to configurational representation of faces. Science, 195, $312-314$.

Chittka, L., \& Dyer, A. (2012). Cognition: Your face looks familiar. Nature, 481, 154 - 155.

Chittka, L., Dyer, A. G., Bock, F., \& Dornhaus, A. (2003). Psychophysics: Bees trade off foraging speed for accuracy. Nature, 424, 388 - 388.

Chittka, L., \& Niven, J. (2009). Are bigger brains better? Current Biology, 19, R995 - R1008. 
de Haan, M., Pascalis, O., \& Johnson, M. H. (2002). Specialization of neural mechanisms underlying face recognition in human infants. Journal of Cognitive Neuroscience, 14, 199 - 209.

Dunbar, R. I. M., \& Shultz, S. (2007). Evolution in the social brain. Science, 317, $1344-1347$.

Dyer, A. G., \& Griffiths, D. W. (2012). Seeing near and seeing far: Behavioural evidence for dual mechanisms of pattern vision in the honeybee (Apis mellifera). Journal of Experimental Biology, 215, 397 - 404.

Dyer, A. G., Neumeyer, C., \& Chittka, L. (2005). Honeybee (Apis mellifera) vision can discriminate between and recognise images of human faces. Journal of Experimental Biology, 208, $4709-4714$.

Dyer, A. G., Rosa, M. G. P., \& Reser, D. H. (2008). Honeybees can recognise images of complex natural scenes for use as potential landmarks. Journal of Experimental Biology, 211, 1180 - 1186.

Dyer, A. G., \& Vuong, Q. C. (2008). Insect brains use image interpolation mechanisms to recognise rotated objects. PLOS ONE, 3, e4086.

Essenberg, C. J., Easter, R. A., Simmons, R. A., \& Papaj, D. R. (2015). The value of information in floral cues: Bumblebee learning of floral size cues. Behavioral Ecology, 26, 1335 - 1344

Farris, S. M. (2008). Structural, functional and developmental convergence of the insect mushroom bodies with higher brains centers of vertebrates. Brain Behavior and Evolution, 72, $1-15$.

Farris, S. M., \& Schulmeister, S. (2011). Parasitoidism, not sociality, is associated with the evolution of elaborate mushroom bodies in the brains of hymenopteran insects. Proceedings of the Royal Society B, 278, 940 - 951.

Giurfa, M., Eichmann, B., \& Menzel, R. (1996). Symmetry perception in an insect. Nature, 382, 458 - 461.

Grangier, J., \& Lester, P. J. (2012). Behavioral plasticity mediates asymmetric competition between invasive wasps and native ants. Communicative \& Integrative Biology, 5, $127-129$.

Gronenberg, W., Ash, L. E., \& Tibbetts, E. A. (2008). Correlation between facial pattern recognition and brain composition in paper wasps. Brain, Behavior and Evolution, 71, $1-14$.

Gross, H. J., Pahl, M., Si, A., Zhu, H., Tautz, J., \& Zhang, S. (2009). Number-based visual generalisation in the honeybee. PLOS ONE, 4, e4263.

Haxby, J. V., Hoffman, E. A., \& Gobbini, M. I. (2002). Human neural systems for face recognition and social communication. Biological Psychiatry, 51, $59-67$.

Horridge, G. A., \& Zhang, S. W. (1995). Pattern vision in honeybees (Apis mellifera): Flower-like patterns with no predominant orientation. Journal of Insect Physiology, 41, $681-688$.

Howard, S. R., Avarguès-Weber, A., Garcia, J., \& Dyer, A. G. (2017). Free-flying honeybees extrapolate relational size rules to sort successively visited artificial flowers in a realistic foraging situation. Animal Cognition, 20, $627-638$

Johnson, M. H., Dziurawiec, S., Ellis, H., \& Morton, J. (1991). Newborns' preferential tracking of face-like stimuli and its subsequent decline. Cognition, 40, $1-19$.

Kanwisher, N. (2000). Domain specificity in face perception. Nature Neuroscience, 3, $759-763$.

Kanwisher, N., McDermott, J., \& Chun, M. M. (1997). The fusiform face area: A module in human extrastriate cortex specialized for face perception. Journal of Neuroscience, 17, $4302-4311$.

Kendrick, K. M., Costa, A. P., Leigh, A. E., Hinton, M. R., \& Peirce, J. W. (2001). Sheep don't forget a face. Nature, $414,165-166$.

Lihoreau, M., Latty, T., \& Chittka, L. (2012). An exploration of the social brain hypothesis in insects. Frontiers in Physiology, 3, 442.

Marzluff, J. M., Miyaoka, R., Minoshima, S., \& Cross, D. J. (2012). Brain imaging reveals neuronal circuitry underlying the crow's perception of human faces. Proceedings of the National Academy of Sciences USA, $109,15912-15917$.

Maurer, D., Le Grand, R., \& Mondloch, C. J. (2002). The many faces of configural processing. Trends in Cognitive Sciences, 6, $255-260$.

Mondloch, C. J., Maurer, D., \& Ahola, S. (2006). Becoming a face expert. Psychological Science, 17, 930 - 934.

Newport, C., Wallis, G., Reshitnyk, Y., \& Siebeck, U.E. (2016). Discrimination of human faces by archerfish (Toxotes chatareus). Scientific Reports, 6, 27523.

Nityananda, V., Skorupski, P., \& Chittka, L. (2014). Can bees see at a glance? Journal of Experimental Biology, 217, $1933-1939$.

Pascalis, O., de Haan, M., \& Nelson, C. A. (2002). Is face processing species-specific during the first year of life? Science, 296, $1321-1323$.

Peterson, M. A., \& Rhodes, G. (2003). Perception of faces, objects, and scenes: Analytic and holistic processes. New York, NY: Oxford University Press.

Rodríguez-Gironés, M. A., Trillo, A., \& Corcobado, G. (2013). Long term effects of aversive reinforcement on colour discrimination learning in free-flying bumblebees. PLOS ONE, 8, e71551. 
Rosenfeld, S. A., \& Van Hoesen, G. W. (1979). Face recognition in the rhesus monkey. Neuropsychologia, 17, 503 509.

Shah, P., Happé, F., Sowden, S., Cook, R., \& Bird, G. (2015). Orienting toward face-like stimuli in early childhood. Child Development, 86, $1693-1700$.

Sheehan, M. J., \& Tibbetts, E. A. (2010). Selection for individual recognition and the evolution of polymorphic identity signals in Polistes paper wasps. Journal of Evolutionary Biology, 23, 570 - 577.

Sheehan, M. J., \& Tibbetts, E. A. (2011). Specialized face learning is associated with individual recognition in paper wasps. Science, $334,1272-1275$.

Srinivasan, M. V. (2010). Honey bees as a model for vision, perception, and cognition. Annual Review of Entomology, $55,267-284$.

Stach, S., Benard, J., \& Giurfa, M. (2004). Local-feature assembling in visual pattern recognition and generalization in honeybees. Nature, $429,758-761$.

Stach, S., \& Giurfa, M. (2005). The influence of training length on generalization of visual feature assemblies in honeybees. Behavioral Brain Research, 161, 8 - 17.

Stejskal, K., Streinzer, M., Dyer, A., Paulus, H. F., \& Spaethe, J. (2015). Functional significance of labellum pattern variation in a sexually deceptive orchid (Ophrys heldreichii): Evidence of individual signature learning effects. PLOS ONE, 10, e0142971.

Stephan, C., Wilkinson, A., \& Huber, L. (2012). Have we met before? Pigeons recognise familiar human faces. Avian Biology Research, 5, $75-80$.

Tanaka, J., \& Gauthier, I. (1997). Expertise in object and face recognition. In P. G. Schyns, R. L. Goldstone, \& D. L. Medin (Eds.), Psychology of learning and motivation, Vol. 36 (83 - 125). San Diego, CA: Academic Press.

Tanaka, J. M., \& Sengco, J. A. (1997). Features and their configuration in face recognition. Memory \& Cognition, 25, $583-592$.

Thompson, E. L., \& Plowright, C. M. (2014). How images may or may not represent flowers: Picture-object correspondence in bumblebees (Bombus impatiens)? Animal Cognition, 17, 1031 - 1043.

Tibbetts, E. A. (2002). Visual signals of individual identity in the wasp Polistes fuscatus. Proceedings of the Royal Society, B, 269, $1423-1428$.

Tibbetts, E. A., \& Dale, J. (2007). Individual recognition: It is good to be different. Trends Ecology \& Evolution, 22, $529-537$.

Tibbetts, E. A., \& Dyer, A. G. (2013). Good with faces. Scientific American, 309, 62 - 67.

Tsao, D. Y., Freiwald, W. A., Tootell, R. B. H., \& Livingstone, M. S. (2006). A cortical region consisting entirely of face-selective cells. Science, 311, $670-674$.

Turati, C., Bulf, H., \& Simion, F. (2008). Newborns' face recognition over changes in viewpoint. Cognition, 106, $1300-1321$.

van Hateren, J. H., Srinivasan, M. V., \& Wait, P. B. (1990). Pattern recognition in bees: Orientation discrimination. Journal of Comparative Physiology, A, 167, 649 - 654.

Warrington, E. K. (1996). Short recognition memory test for faces. Windsor, UK: Psychology Press.

Wolf, S., Roper, M., \& Chittka, L. (2015). Bumblebees utilize floral cues differently on vertically and horizontally arranged flowers. Behavioral Ecology, 26, $773-781$.

Wu, W., Moreno, A. M., Tangen, J. M., \& Reinhard, J. (2012). Honeybees can discriminate between Monet and Picasso paintings. Journal of Comparative Physiology A, 199, 45 - 55.

Wystrach, A., \& Beugnon, G. (2009). Ants learn geometry and features. Current Biology, 19, 61 - 66.

Yin, R. K. (1969). Looking at upside-down faces. Journal of Experimental Psychology, 81, 141 - 145.

Young, A. W., Hellawell, D., \& Hay, D. C. (1987). Configurational information in face perception. Perception, 16, $747-759$.

Zhang, S., Srinivasan, M. V., Zhu, H., \& Wong, J. (2004). Grouping of visual objects by honeybees. Journal of Experimental Biology, 207, 3289 - 3298.

Zhen, Z., Fang, H., \& Liu, J. (2013). The hierarchical brain network for face recognition. PLoS ONE, 8, e59886. 